\title{
Three-Dimensional Particle Tracking in Microfluidic Channel Flow Using In and Out of Focus Diffraction
}

\author{
Bushra Tasadduq ${ }^{1}$, Gonghao Wang ${ }^{1}$, Mohamed El Banani ${ }^{1}$, Wenbin Mao ${ }^{1}$, Wilbur Lam ${ }^{2}$, \\ Alexander Alexeev ${ }^{1}$, and Todd Sulchek ${ }^{1,2}$ \\ ${ }^{1}$ Woodruff School of Mechanical Engineering, Georgia Institute of Technology, Atlanta GA, 30332, USA \\ ${ }^{2}$ Wallace H. Coulter Department of Biomedical Engineering, Georgia Institute of Technology, Atlanta GA, 30332, \\ USA
}

\begin{abstract}
Three-dimensional particle tracking is important to accurately understand the motion of particles within complex flow fields. We show that three-dimensional trajectories of particles within microfluidic flow can be extracted from two-dimensional bright field video microscopy. The method utilizes the defocusing that occurs as particles move out of the objective focal plane when viewed through a high numerical aperture objective lens. A fast and simple algorithm based on cross correlation to a set of reference images taken at prescribed amounts of defocus is used to extract out-of-plane particle position. In-plane particle position is determined through center point detection and therefore the particle position in all three dimensions can be constructed at each time point. Particle trajectories at high flow velocity of greater than $2 \mathrm{~mm} / \mathrm{s}$ can be tracked by utilizing a high speed camera to obtain unblurred images. Three dimensional computational fluid simulations are used to validate the particle tracking methods.
\end{abstract}

Keywords: Particle tracking, microfluidics.

\section{Introduction:}

Conventional video microscopy depicts particle motion projected onto a plane defined by the focus of the objective. For many applications, visualization of motion in the third dimension is required to fully understand the trajectory of particles. Three-dimensional tracking particularly benefits visualizing particle motion in complex flow fields such as that in biomedical devices (Wu, Di Carlo et al. 2008, Tsai, Kita et al. 2012, Wang, Mao et al. 2013, Wang, Crawford et al. 2015) and physiological systems. Several methods can be used to track particles in three dimensions. Laser Doppler velocimetry (Weinberg 1969) utilizes the wavelength shifts due to particle motion within the path of multiple lasers to reconstruct particle motion. Particle image velocimetry (PIV) measures the position and velocity of particles by pulsing a laser and observing the illuminated locations with microscopy (Melling 1997). Extending this technique, two cameras can be positioned along two Cartesian axes to track particles along all three dimensions (Maas, Gruen et al. 1993, Cheung, Ng et al. 2005). Such systems can be complex to implement, however, and require careful alignment of the optical components which results in reduced observational volume that is limited by the optical depth of field in two dimensions. Recently, a single camera combined with multiple objectives was used to track particles in threeCorresponding author

E-mail address: todd.sulchek@me.gatech.edu

Phone number: 650-954-4592

(C) 2015. This manuscript version is made available under the Elsevier user license

http://www.elsevier.com/open-access/userlicense/1.0/ 
dimensions (E. Toprak 2007). Single camera systems can also observe changes in the particle zposition through out-of-focus motion with respect to a fixed position, such as the water-glass interface (Deng 2009), though this technique may be prone to aberrations in z-motion for large out-of-plane particle motion.

We present an accurate and simple method for three-dimensional particle tracking within microfluidic channel flow that utilizes a single objective and a single high-speed camera. The method combines standard two-dimensional particle tracking from video (Salman S Rogers 2007) and a correlation algorithm that determines out-of-focus particle motion to additionally compute the third dimension. The out-of-focus correlation algorithm utilizes the contrast change and incremental changes in blurriness of the particle image that occurs as the particle moves in relation to the focal plane, as demonstrated in studies of particles actuated with a piezo-driven stage (Zhang 2008). We validate the algorithm for measuring particle out-of-plane motion flowing in a microfluidic channel by utilizing cross correlation of the particles to a set of reference images of a known position. To improve the resolution of the z-axis motion, interpolation of the reference images was implemented to achieve submicron resolution. We utilize these methods to track the three dimensional trajectories of particles flowing at high speeds through a microfluidic channel.

_ENREF_14

\section{Experimental Setup:}

Spherical polystyrene particles of $3 \mu \mathrm{m}, 4 \mu \mathrm{m}$, and $6 \mu \mathrm{m}$ diameters (Bangs Laboratories Inc.) were used in the flow experiments. Dispersions were prepared in deionized water with a concentration of approximately 2 particles $/ \mu \mathrm{L}$. The particle solution was contained in a syringe and mounted on a syringe pump (PHD 2000 Harvard Apparatus), which infused the suspension at a controlled flow rate $(0.0001-0.05 \mathrm{~mL} / \mathrm{min})$ through capillaries connected to a microfluidic channel. The microchannel was formed from replica molded polydimethyl siloxane (PDMS) on a permanent mold made with SU8, and plasma bonded to a glass slide, as described previously (Wang, Mao et al. 2013). The microfluidic channel featured periodical diagonal ridges that generate a helical three dimensional flow field, as demonstrated previously by several research groups (Dertinger 2002, Choi 2007, Alexeev 2011, Wang, Mao et al. 2013). The experimental setup is shown in Figure 1. The microfluidic chip was mounted on an inverted microscope (Nikon Eclipse Ti). Particle trajectories were captured with a 40X objective and a high-speed camera (Phantom V7.3 Vision Research). In order to accurately record the trajectories of particles, high resolution (1150 by 1150 pixels) and high frame rate ( $\geq 3000 \mathrm{fps}$ ) videos were recorded for analysis.

\section{Particle Tracking Algorithm:}

\subsection{Lateral Position Algorithm:}

The two-dimensional image analysis tool is based upon the open source code PolyParticle Tracker optimized for noisy backgrounds (Salman S Rogers 2007). A full description of the lateral position determination was described previously (Salman S Rogers 2007). Each frame of 
the video was extracted through MATLAB routine and the image was smoothed to remove discretization noise by convolution with a Gaussian function. The convolution is given in terms of image intensity $I(x, y)$ by the following equation:

$$
I(x, y)=\sum_{i=-w}^{w} \sum_{j=-w}^{w} I_{r a w}(x+i, y+j) e^{\frac{-i^{2}+j^{2}}{4 \lambda^{2}}},
$$

in which $2 w+1$ is the extent of the boxcar averaging used to model background in each frame and is an integer larger than a single sphere's apparent radius in pixels, but smaller than an intersphere separation. The correlation length, $\lambda$, is set to 1 , and $w=3 \lambda$ in order to sufficiently approximate the unbounded convolution. The local maximum or minimum in $I(x, y)$ determines the position of the particle $\left(x_{0}, y_{0}\right)$ in each extracted image. For each extrema, the adjacent points of inflexion $\left(x_{0}, y\right)$ and $\left(x, y_{0}\right)$ were found. The initial radius $R_{0}$ is estimated as the mean distance to these four points of inflexion. To determine resolution below the pixel dimensions, a sub-pixel refinement was utilized (Salman S Rogers 2007) with estimated position $\left(x_{0}, y_{0}\right)$ and radius $R_{0}$ by fitting the intensity map of the image $I(x, y)$ defined as:

$$
I_{f i t}(x, y)=\sum_{i=0, j=0}^{i+j=4} P_{i j} x^{i} y^{i}
$$

The fit was weighted at each pixel by the Gaussian function:

$$
W(x, y)=\exp \frac{-\alpha\left(x^{2}+y^{2}\right)}{R^{2}},
$$

in which $R$ is the estimated radius and $\alpha$ is the decay length set to the radius of the particle, which was determined from the image. The particle center was determined by the local maximum of the polynomial fit. A polynomial fit offered several advantages. Firstly, the fit is not sensitive to the edges of a particle but most sensitive to pixels well within the borders of the particle image. Secondly, the fit is optimized to identify spherical or elongated shapes. The third advantage is that, unlike the previous methods, the procedure of determining the particle center is inherently insensitive to the image background (Salman S Rogers 2007). In order to link particle position from multiple frames to create a trajectory, the coordinates of a particle in the preceding frame $\mathrm{T}$ are used as the initial estimate for its position in the current frame $\mathrm{T}+1$, provided that the particle does not move further than its own radius from $\mathrm{T}$ to $\mathrm{T}+1$. The frame rate should therefore be selected appropriately. 


\subsection{Axial Position Estimation Algorithm:}

The images of a micrometer-scale particle appear to be very different when out-of-focus. As shown in Figure 2, the in-focus image of a microparticle has sharp edges with high contrast, while the out-of-focus image is diffuse with concentric diffraction rings. In general, the abovefocus images are distinct due to the inherent image aberrations caused by the objective lens (Deng 2009). Therefore, once the relationship between the off-focus image of the particle and its axial position is established, it is possible to accurately determine the axial positions of the particle from the acquired off-focus images. We determined the out-of-plane position of each particle by the degree of defocusing of the particle (Lewis 1994), evaluated by a correlation algorithm comparing the image to a set of reference images of a similar particle at specified amounts of defocusing. The estimation of the axial position using degree of defocus involves three steps. First, prior to each flow experiment we generate reference images and interpolated images. A total of 13 reference images are generated with a step size of $1 \mu \mathrm{m}$ and interpolated images are computed with a resolution of $0.01 \mu \mathrm{m}$. Second, after the flow experiment each video frame is analyzed to determine the $2 \mathrm{D}$ (lateral) position of each particle. Third, the axial position of each particle is determined by comparing the image from each frame to the calibrated defocused images using a matching algorithm. To generate references images, particles were affixed to the bottom of the microfluidic channel and images were taken while changing the focal plane in $1 \mu \mathrm{m}$ step sizes. Hence, the reference image scale includes a single particle in focus and various degrees of out of focus by calibrated adjustment of the microscope objective. The set of reference images used in these experiments are shown in Figure 2.

To increase the resolution of the particle z-height, we utilized interpolation between adjacent reference images. The reference images were recorded with $1 \mu \mathrm{m}$ spacing. We generate additional interpolated reference images as shown in Figure 3. Images are formatted in MATLAB as a 3D array $(M x N x 3)$ where $\mathrm{M}, \mathrm{N}$ are the dimensions of the image and 3 layers for Red, Green and Blue layers. Values for each color layer of each pixel span 0 to 255. Starting with a set of images of the same particle at different depths (with the particle having the same position in each image, and that position being the center), and assuming that the change in the focus of the particles is continuous, we set the value of each pixel to be a function of the depth so that we have $3 * M * N$ functions. Using the values of the pixels in the given set of images as initial values and applying spline interpolation to each function, we interpolate the value of each pixel at any given depth within the range of depths of the set of the reference images. The interpolated values form an array which is then used to create the interpolated image. Selected interpolated images were compared with the original reference images and we found the relative error to be less than $2 \%$ using the MATLAB routine imshowpair. Figure 3 shows the interpolated images between reference images for $1 \mu \mathrm{m}$ and $2 \mu \mathrm{m}$ focal points, with a step size of $0.1 \mu \mathrm{m}$. When comparing a particle to the reference, the image was automatically cropped and centered to accurately determine the cross correlation coefficient. The center of the particle is determined using three points on the circumference and assuming circularity (French 1911).

The degree of defocus is also related to the number and thickness of concentric diffraction rings of the three dimensional point spread function, which accounts for uniqueness of the cross correlation approach. We utilized this feature of defocused images of microparticles to obtain a z-position scale. This scale can then be compared to a particle of interest to determine its zposition through a cross-correlation analysis. The cross-correlated algorithm was implemented 
using a MATLAB routine based on previous studies (Lewis 1994). We take [ $\left.\left(x_{1}, y_{1}\right),\left(x_{2}, y_{2}\right), \ldots . .\left(x_{N}, y_{N}\right)\right]$ and $\left[\left(a_{1}, b_{1}\right),\left(a_{2}, b_{2}\right), \ldots . .,\left(a_{N}, b_{N}\right)\right]$ as the pixel coordinates for analyzed image and reference image respectively, in which $N^{2}$ represents the feature size. The cross-correlation output matrix is calculated as a double matrix with [ $\left.\left(x_{1}+a_{1}, y_{1}+b_{1}\right),\left(x_{2}+a_{2}, y_{2}+b_{2}\right), \ldots,\left(x_{N}+a_{N}, y_{N}+b_{N}\right)\right]$ pixel coordinates with $\left[c_{1}, c_{2}, \ldots ., c_{N}\right]$ corresponding correlation coefficients. From this output matrix we extract $x$ and $y$ pixel coordinates which correspond to the maximum value of correlation coefficient. This maximum value determines the relative likeness of the particle of interest and the reference image in each frame. The implementation closely follows following formula from (Lewis 1994) and (Haralick 1992):

$$
\gamma(u, v)=\frac{\sum_{x, y}\left[f(x, y)-\bar{f}_{u, y}\right][t(x-u y-v)-\bar{t}]}{\left\{\sum_{x, y}\left[f(x, y)-\bar{f}_{u, v}{ }^{2} \sum_{x, y}[t(x-u y-v)-\bar{t}]^{2}\right\}^{0.5}\right.}
$$

in which $f=\left[\left(x_{1}, y_{1}\right),\left(x_{2}, y_{2}\right), \ldots \ldots\left(x_{N}, y_{N}\right)\right]$ and $\bar{t}$ is the mean of $\mathrm{t}=\left[\left(a_{1}, b_{1}\right),\left(a_{2}, b_{2}\right), \ldots \ldots,\left(a_{N}, b_{N}\right)\right]$ as the pixel coordinates for analyzed image and reference image respectively, in which $N^{2}$ represents the feature size. $\bar{f}_{u, v}$ is the mean of $f(x, y)$ in the region under the reference image template.

\section{Results and Discussion:}

For this study the microchannel with the top of the channel decorated with diagonal ridges generates helical recirculation and lateral pressure gradients (Figure 1), which results in helical trajectories of particles, as shown by experiments (Dertinger 2002, Choi 2007, Wang, Mao et al. 2013) and simulation (Alexeev 2011).

Videos of particles flowing in the microchannel were recorded (Supplemental Video 1). Still frames at different time points in the trajectory are shown overlaid in Figure 4. The particle $x$ and $y$ coordinates for each time point were calculated as described in the methods. The $z$ coordinate for each time point was determined through calculation of the cross correlation coefficient to the set of defocused reference images, as described in the methods. The calculation of the cross correlation coefficient of a particle from a typical still frame is shown in Figure 5. The z-position at each time point was combined with the calculated $x$ and $y$ coordinate to generate a three dimensional position. The analysis continued for all frames in the video to obtain a full threedimensional trajectory for particles of various sizes.

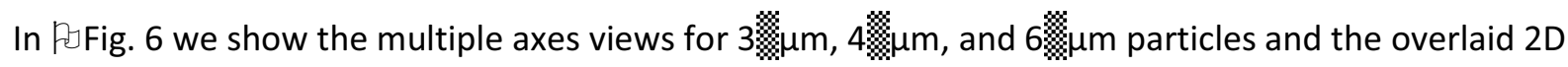
video frames. As expected from previous studies, we observe a sinusoidal motion in $x-y$ plane and the $x$ - 
z plane (Dertinger 2002, Choi 2007, Alexeev 2011, Wang, Mao et al. 2013). The three dimensional visualization of the trajectory indicates helical motion. Previous studies have indicated that the trajectory should be particle-size dependent, in which larger particles having smaller magnitudes of $\mathrm{y}^{-}$

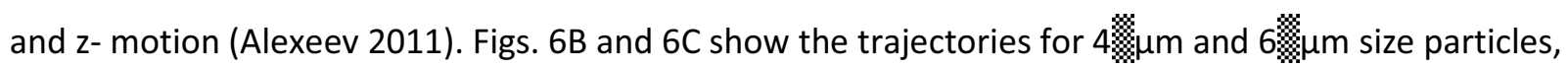
respectively. Examining the $x-y$ trajectories of particles with different sizes, we notice the $\Delta y /$ ridge for the particles varies inversely with particle size, as expected from published reports (Dertinger 2002).

This is further confirmed in Fig. 6D which shows the calculated $\Delta y /$ ridge and $\Delta z /$ ridge for different size particles. poFig. 7 shows cross-stream velocity distribution in a channel with ridged walls. Note that the diagonal ridges create circulatory flows that transport fluid to the left in the channel midplane and to the right near the top and bottom walls (Alexeev 2011). Hence, the methods presented in this work can be used to better understand how microfluidic flow can be utilized for particle size separation (Choi 2007, Alexeev 2011). To further validate the tracking results, we compare our measured trajectory to that obtained by previously validated computational fluid dynamics simulations (Alexeev 2011). Pofig. 8 shows the comparison of z-positions of simulated and experimental results of a particle flowing within the channel. The simulations fully considered the geometry of the device used, the size of the particles, and the flow rates used in the experiment. The results show that the tracked z-positions closely match the computationally expected path.

The relative error between the experimental and simulated trajectories was calculated and shown in Figure 9. Sources of error of the method can include variations in illumination, the optical variability of the medium, and features of the microchannel background. The error is found to be maximum where the particles are traversing under a microchannel ridge (x position range: $25 \mu \mathrm{m}$ to $50 \mu \mathrm{m}$ ). This is due to the presence of channel features in the frames that are not present in the calibrated reference images. One simple solution to remove this error is to utilize interpolation between the points where particle enters and leaves a ridge. Overall, the average error is $25 \%$ which corresponds to a maximum of $1 \mu \mathrm{m}$ absolute error for all three sizes and compares favorably to some other 3D tracking techniques in which the reported absolute error is $4 \mu \mathrm{m}(\mathrm{C}$. Snoeyink 2013). We observed that all the particle trajectories we manually examined closely followed the observed trajectories, a criterion for accurate particle tracking (E. Meijering February 2012). Other sources of error include the estimation errors due to spatial sampling and noise (Zhang 2008). In the future we can improve the performance of the algorithm by using smaller step sizes in the generated reference images.

We utilize a method described previously (Zhang 2008) to calculate the minimum limit of the bead size we can track using current setup and sampling frequency in order to reconstruct the image without any loss of information. The point spread function (PSF) of the microscope dictates the upper limit to the frequency spectrum of the image and hence a detector samples the PSF at or above the Nyquist frequency so that there will be no information loss when sampling the image of a bead of any size. The PSF, in-focus plane takes the shape of an Airy disc for an aberration-free objective lens. The Airy disc has a diameter of $d_{a}=1.22 * \lambda /$ Numerical Aperture $=1864 \mathrm{~nm}$ in our current setup, where $\lambda=550 \mathrm{~nm}$ is used as the central wavelength of the white light source and the numerical aperture is 0.36 is the average 
value of objective (CFI60, 0.2) and condenser (LWD, 0.52) numerical apertures of Nikon Ti microscope. The spectrum of the Airy disc intensity profile is analyzed and the sampling frequency of $/ 1 / d_{a}$ is determined as the minimal frequency to sample the Airy disc without aliasing. The sampling width needs to be smaller than $1864 / 1=1864 \mathrm{~nm}$ to sample the PSF. In our current system, the sampling width, the sampling plane is

Pixel size / Magnification factor $=22 \mu \mathrm{m} / 40=550 \mathrm{~nm}$ is much smaller than the specified range.

When the bead diameter $d B$ is larger than $d_{a}$ the image spectrum will be limited by the spectrum of the object rather than the PSF. With a similar analysis, the smallest bead size that can be measured using our current setup can be calculated by equalizing the required minimal sampling frequency $1 / d B$ and current sampling frequency $1 / 550 \mathrm{~nm}$, and the result is $d B=$ $550 \mathrm{~nm}$.

This method also has limitations regarding large changes in vertical motion. The particles far from the focal plane cannot be accurately detected by the objective and hence this method cannot be used in such cases. Additionally, features in the channel, such as the diagonal ridges, can produce anomalous results in calculation of the cross correlation coefficient. Therefore the determination of the z-position may be inaccurate when the particle is obstructed by the ridge. Interpolation can be used to plot approximate position of the beads when they are flowing under the ridge. We observed that the out of focus diffraction is symmetric whether we move above or below the focal plane, hence we are limited to focusing at one extreme of the channel bottom and record particles flowing on one side of the out of focus diffraction curve.

\section{Conclusion:}

From two-dimensional bright field videos of particle flow, we are able to quantitatively determine the three dimensional trajectories of motion. The fast and simple procedure utilizes changes in the particle image as it moves in relation to the focal plane. The applications of this method are quite versatile and can be used to understand complex microfluidic flow of particles and other spherical particles such as cells.

\section{Acknowledgments}

This research was supported by NSF project number CBET-0932510, the Center for Pediatric Nanomedicine Seed Grant, the Fulbright Scholarship Program, and the President's Undergraduate Research Award (PURA) program at Georgia Tech. 


\section{Figures captions:}

Figure 1: Experimental setup showing the microfluidic devices placed onto the microscope and a schematic of particles flowing through the device. The video frames are captured using a high speed camera and displayed on a computer.

Figure 2: Reference images taken with different focal points for a $4 \mu \mathrm{m}$ particle. Cross correlation coefficients are calculated between the particle images at known z-positions and the reference images. The $z$-position of the particle can thus be determined from the degree of defocus and is also related to the number and thickness of concentric diffraction rings.

Figure 3: Utilizing two reference images at the focal points $1 \mu \mathrm{m}$ and $2 \mu \mathrm{m}$, a set of interpolated images can be generated with a step size of $0.1 \mu \mathrm{m}$.

Figure 4: A schematic of the algorithm to perform three dimensional particle tracking. Video frames are captured and used to identify particles and determine their $x$ and $y$ coordinates using polynomial fitting with Gaussian weight. The image of the particle is cropped and then a cross correlation coefficient is generated against the reference images to determine the $z$ height of the particle. The process is iterated for each time point in the video to generate a three dimensional trajectory.

Figure 5: The determination of the $z$ height is tested by calculating the cross correlation coefficient between a particle at an unknown $z$-position and the reference images.

Figure 6: Experimentally calculated trajectories during one period of motion between ridges for A) $3 \mu \mathrm{m} \mathrm{B)} 4 \mu \mathrm{m}$ and C) $6 \mu \mathrm{m}$ particles. D) Data extracted from the computed trajectories show that different size beads move with different amplitudes in $\mathrm{z}(\Delta \mathrm{z} /$ ridge) and show different amounts of transverse motion ( $\Delta y /$ ridge). The size dependence of $\Delta y /$ ridge can be utilized for size-based separation of particles.

Figure 7: Cross-stream (transverse) velocity distribution within a channel with ridged walls, which result in particles with helical flow trajectories.

Figure 8: Comparison of the $x-z$ experimental trajectories with the computationally simulated trajectories for different size particles.

Figure 9: The relative error between the experimental and simulated trajectories during a typical trajectory, with the typical error between $20 \%-25 \%$.

Supplemental Video: Videos were recorded with microfluidic chip mounted on an inverted microscope (Nikon Eclipse Ti) utilizing a 40X objective and equipped with a high speed camera (Phantom V7.3 Vision Research). In order to accurately capture the trajectories of particles, high resolution (1150 by 1150 pixels) and high frame rate $(\geq 3000 \mathrm{fps})$ videos were recorded for analysis. The videos were recorded with flow rate of $0.0001 \mathrm{ml} / \mathrm{min}$.

\section{References}


Alexeev, M. W. a. (2011). "A. Continuous sorting of microparticles by size in ridged microchannels." Physics of Fluids.

C. Snoeyink, S. W. (2013). "A novel 3D3C particle tracking method suitable for microfluidic flow measurements." Experiments in fluids 54(1).

Cheung, K., et al. (2005). "Three dimensional tracking of particles and their local orientations." Flow Meas Instrum 16: 8.

Choi, S. a. J. K. P. (2007). "Continuous hydrophoretic separation and sizing of microparticles using slanted obstacles in a microchannel." Lab on a Chip 7(7): 8.

Deng, Y. a. S., J. W. (2009). "Effect of aberration on height calibration in three-dimensional localizationbased microscopy and particle tracking." Applied Optics 48: 5.

Dertinger, A. A., I. Mezic ,H. A. Stone, and G. M. Whitesides (2002). "Chaotic mixer for microchannels." Science 295(5555): 5.

E. Meijering, O. D. I. S. (February 2012). Methods for Cell and Particle Tracking In Imaging and Spectroscopic Analysis of Living Cells. Methods in Enzymology. P. M. Conn, Elsevier. 504: 18.

E. Toprak, H. B., B. H. Blehm, and P. R. Selvin (2007). "Three-dimensional particle tracking via bifocal imaging." Nano Lett. 7(7): 3.

French, T. E. (1911). A Manual of Engineering Drawing for Students and Draftsmen New York, McGraw-Hill.

Haralick, R. M., and Linda G.Shapiri (1992). Computer and Robot Vision, Addison-Wesley II: 316-317.

Lewis, J. P. (1994). "Fast template matching." Vision Interface '95, Proceedings: 120-123.

Maas, H. G., et al. (1993). "Particle tracking velocimetry in three- dimensional flows." Experiments in Fluids 15(2): 14.

Melling, A. (1997). "Tracer particles and seeding for particle image velocimetry." Meas. Sci. Technol. 8: 11.

Salman S Rogers, T. A. W., Xiubo Zhao and Jian R Lu. (2007). "Precise particle tracking against a complicated background: polynomial fitting with Gaussian weight." Physical Biology 4: 8. 
Tsai, M., et al. (2012). "In vitro modeling of the microvascular occlusion and thrombosis that occur in hematologic diseases using microfluidic technology." The Journal of Clinical Investigation 122(1): 11.

Wang, G., et al. (2015). "Microfluidic cellular enrichment and separation through differences in viscoelastic deformation." Lab on a Chip.

Wang, G., et al. (2013). "Stiffness Dependent Separation of Cells in a Microfluidic Device." PLoS ONE $\mathbf{8}(10)$.

Weinberg, M. J. R. S. a. F. J. (1969). "The Measurement of Velocity by Applying SchlierenInterferometry to Doppler-Shifted Laser Light." Proceedings of the Royal Society of London. Series A, Mathematical and Physical Sciences 311(1506): 8.

Wu, L. Y., et al. (2008). "Microfluidic Self-Assembly of Tumor Spheroids for Anti-Cancer Drug Discovery." Biomedical Microdevices 10(2): 6.

Zhang, Z. a. M. C. H. (2008). "Three-Dimensional particle tracking with subnanometer resolution using off-focus images." Applied Optics 47(13): 10.

\section{HIGHLIGHTS:}

- From two-dimensional bright field videos of particle flow, we are able to quantitatively determine the three dimensional trajectories of motion.

- The method is simple as only single camera is used to perform the three dimensional tracking.

- The algorithm can be used to understand complex microfluidic flow of particles and other spherical particles such as cells. 


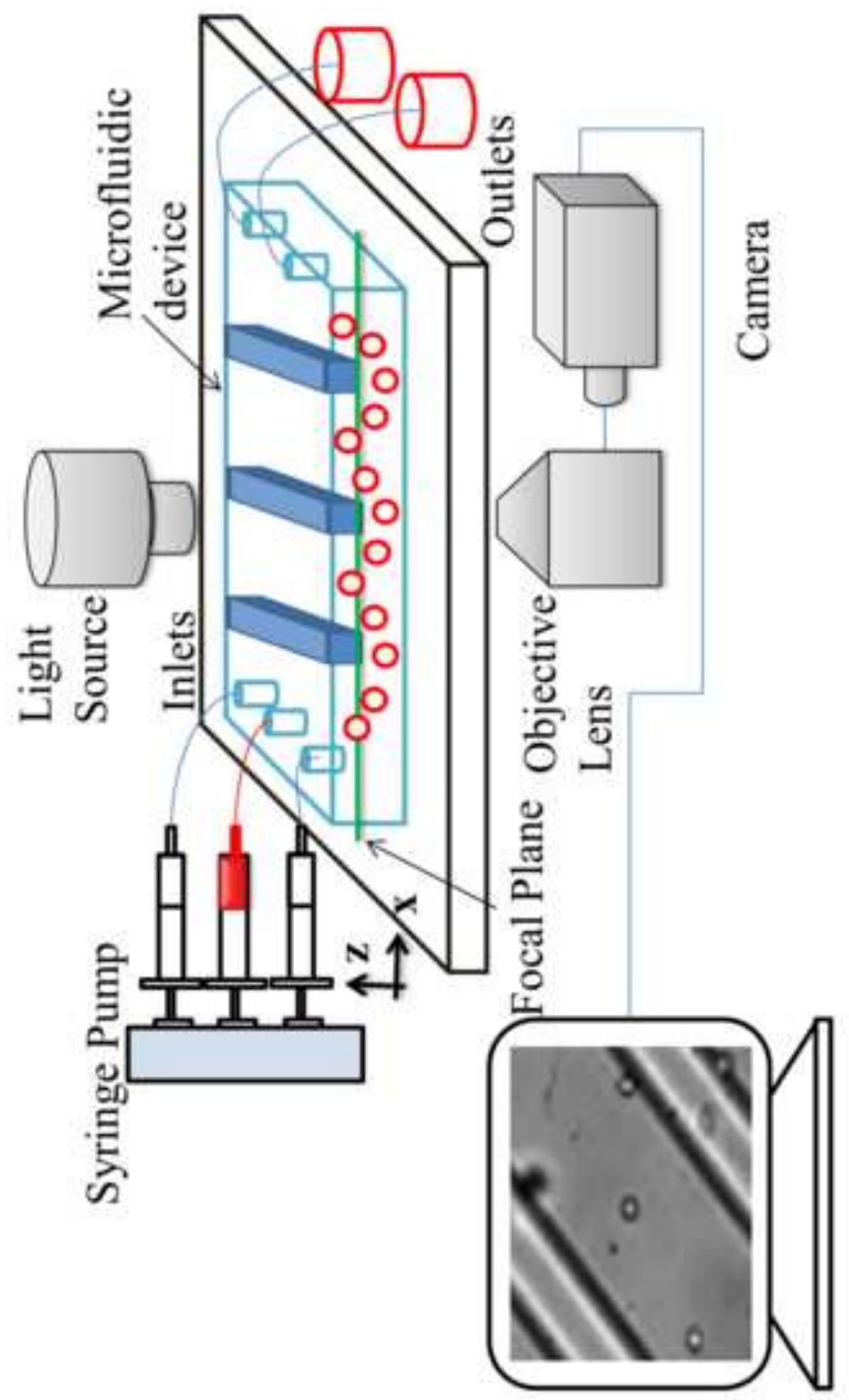




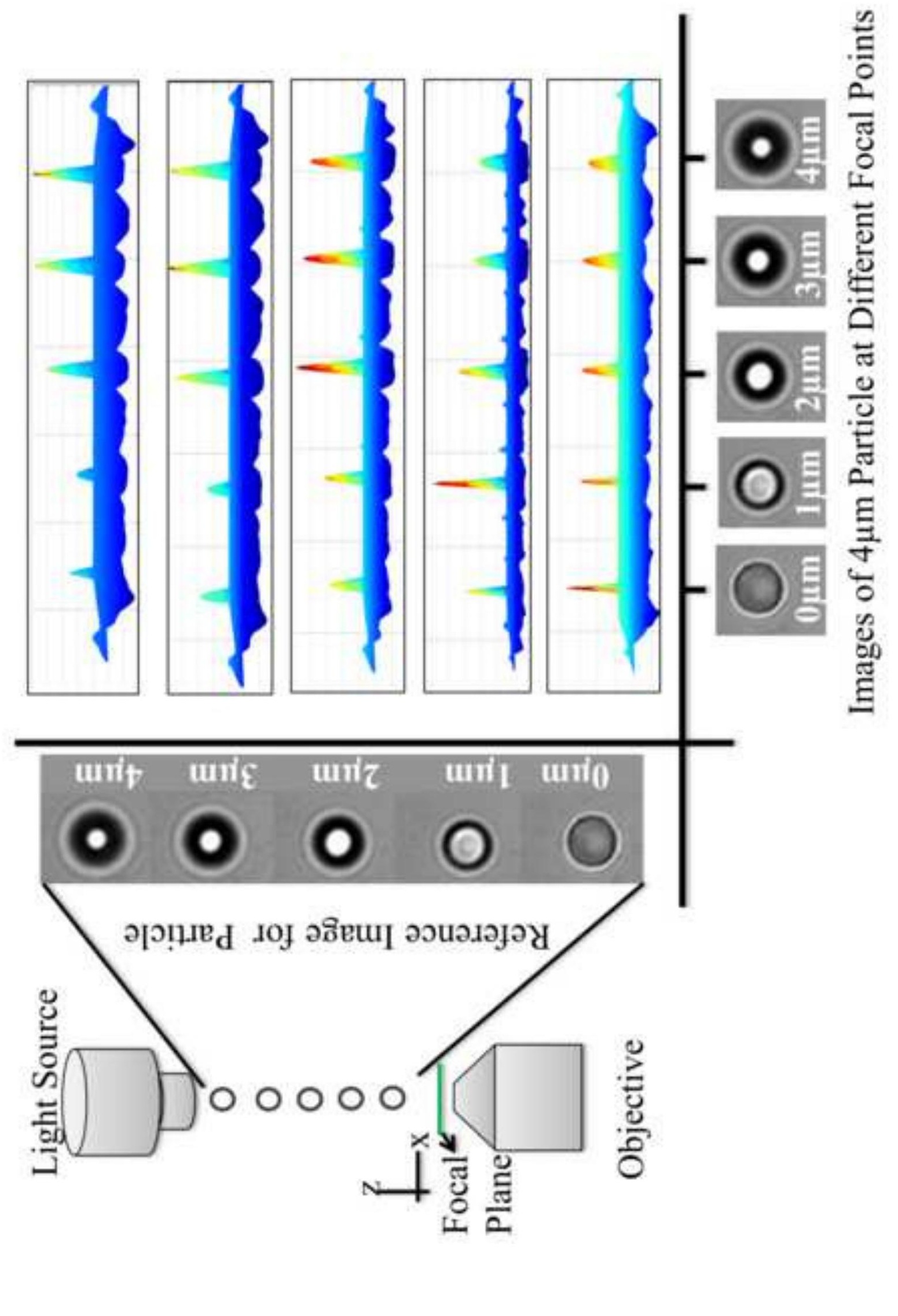




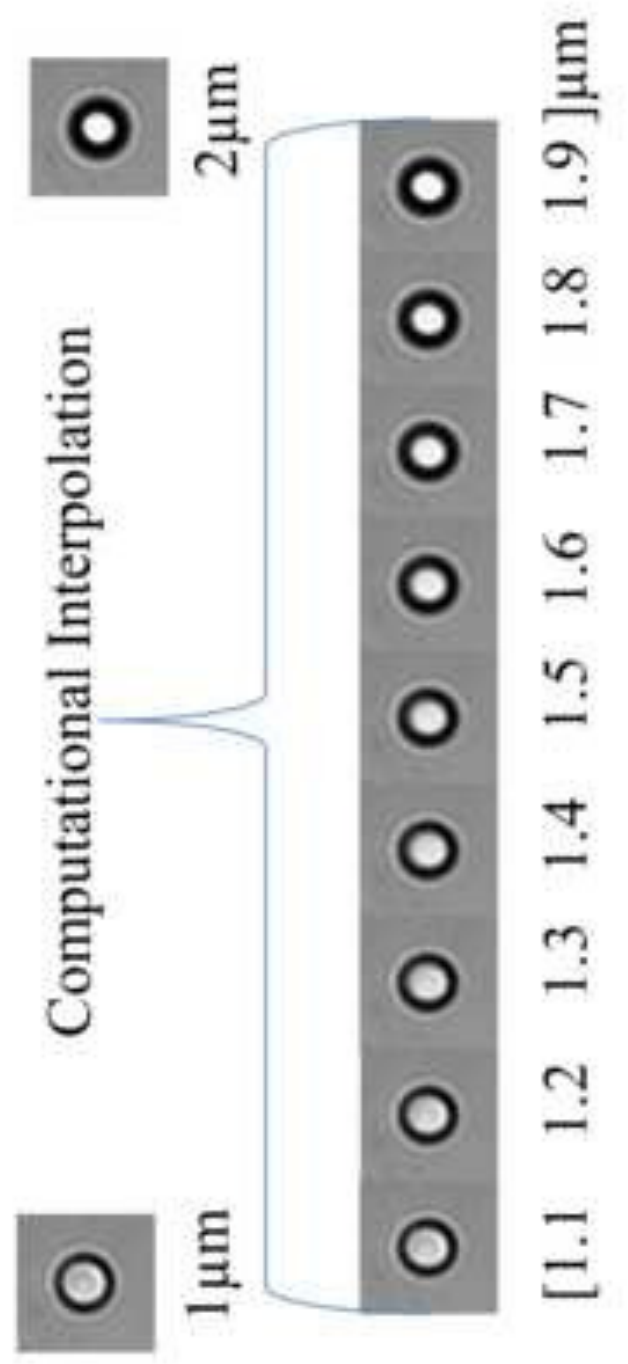

꿍 

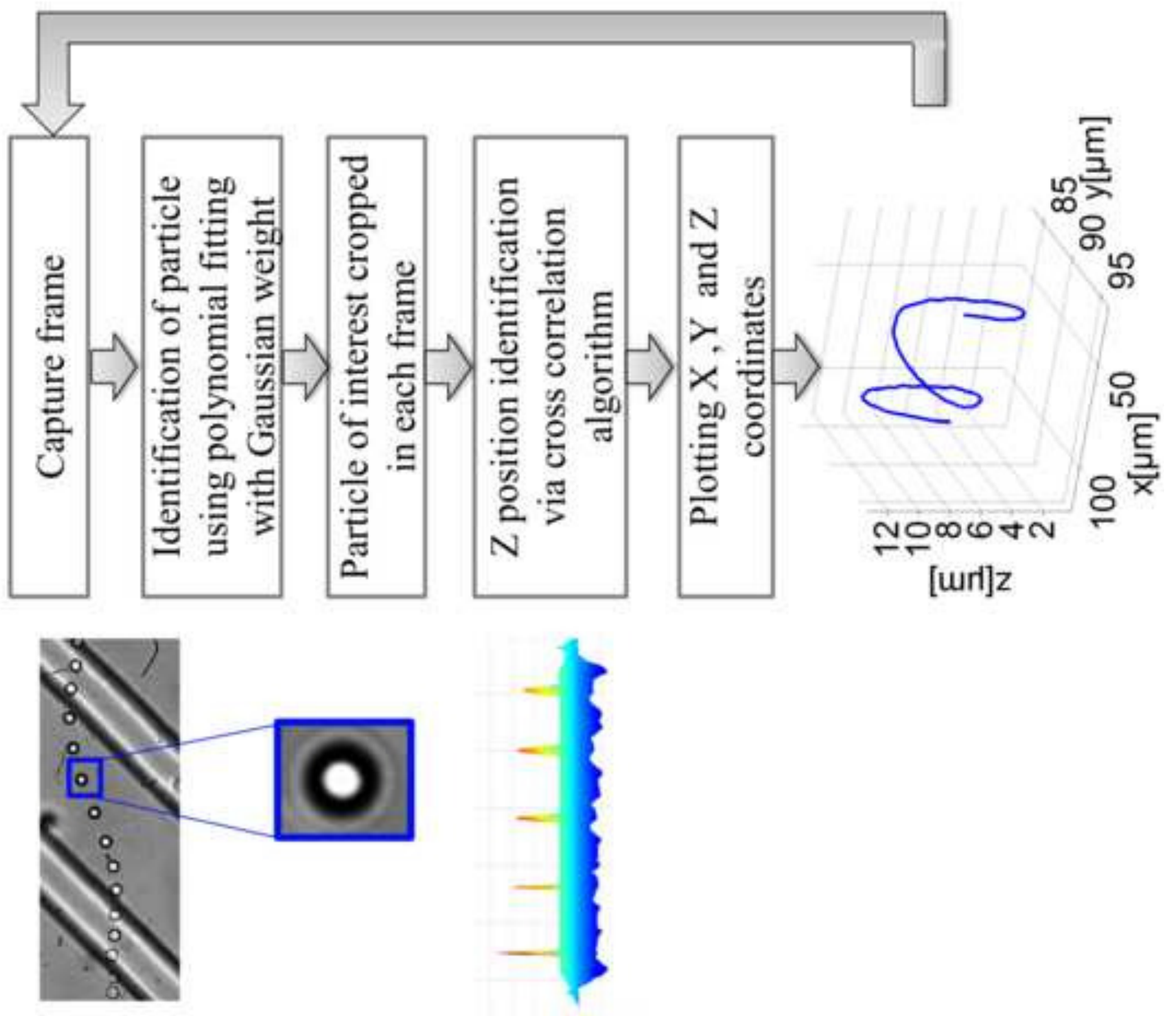


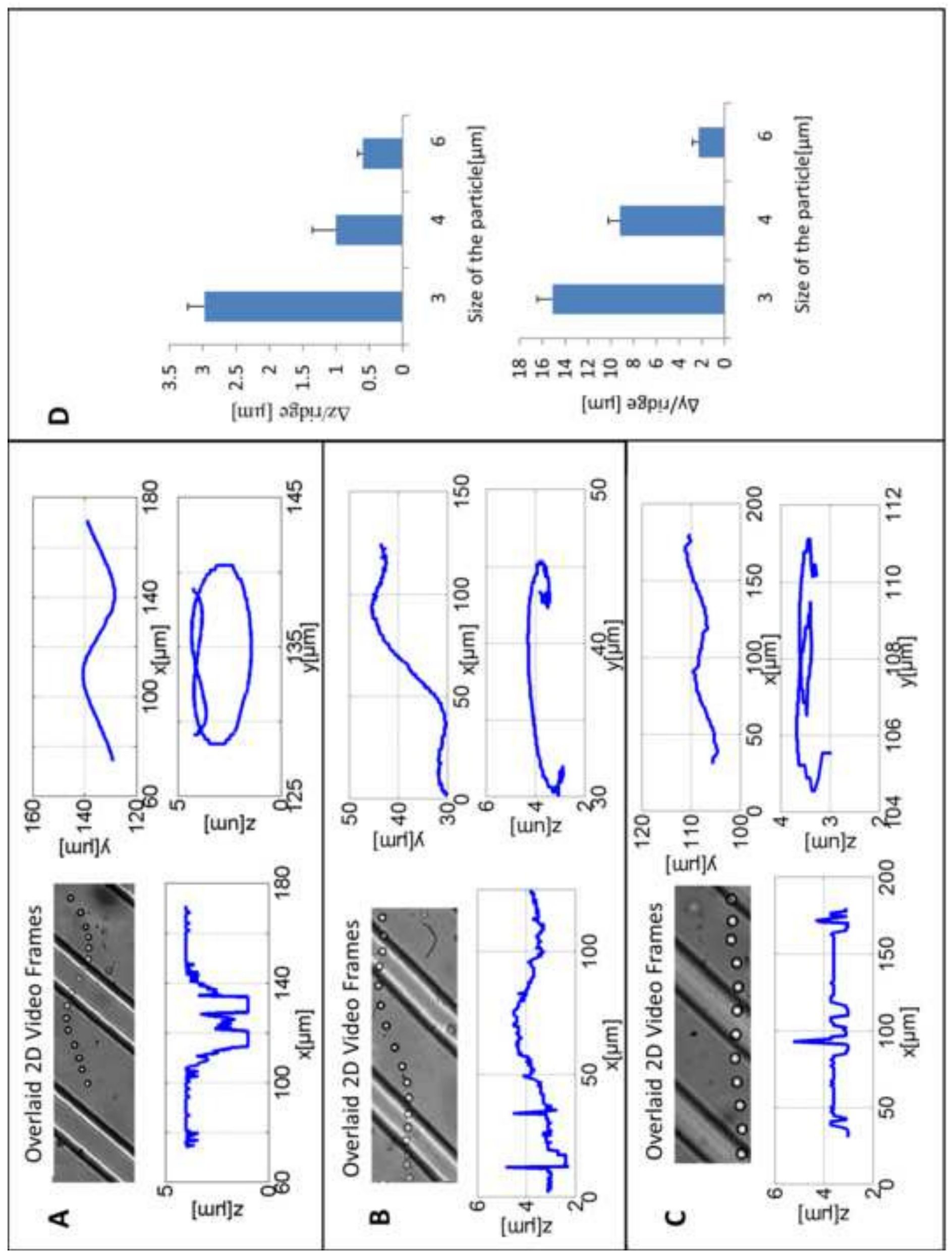




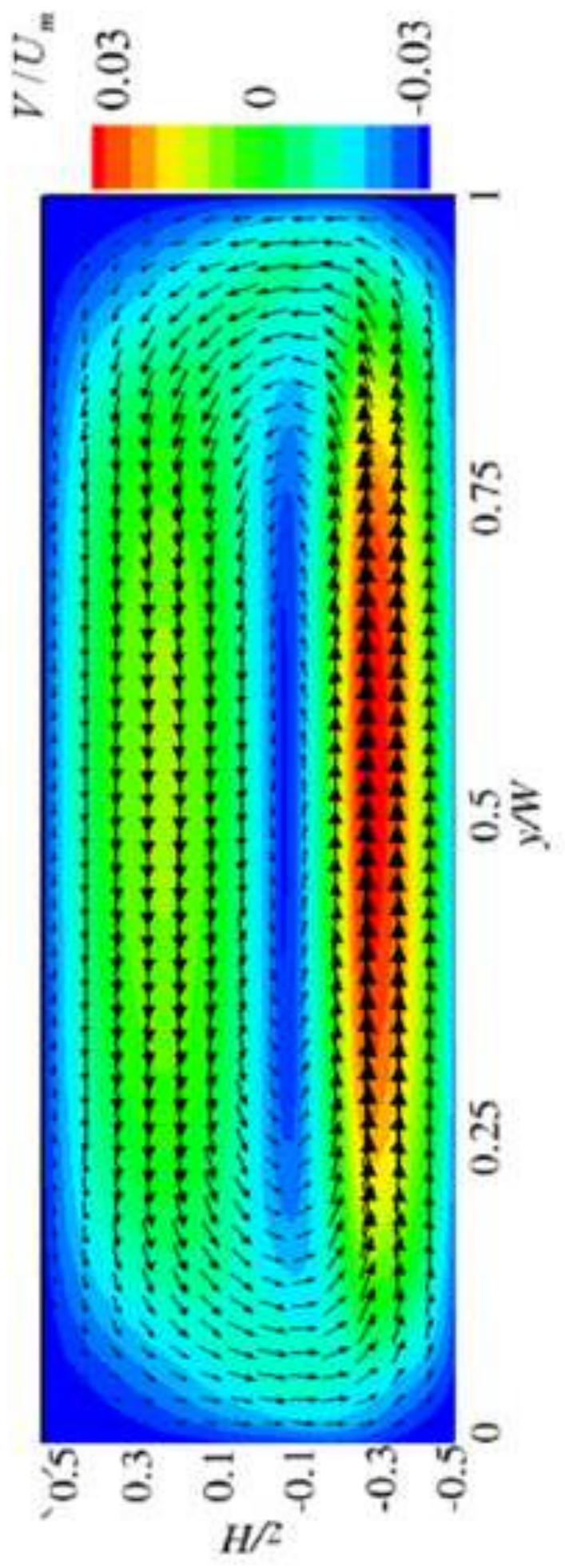

손 

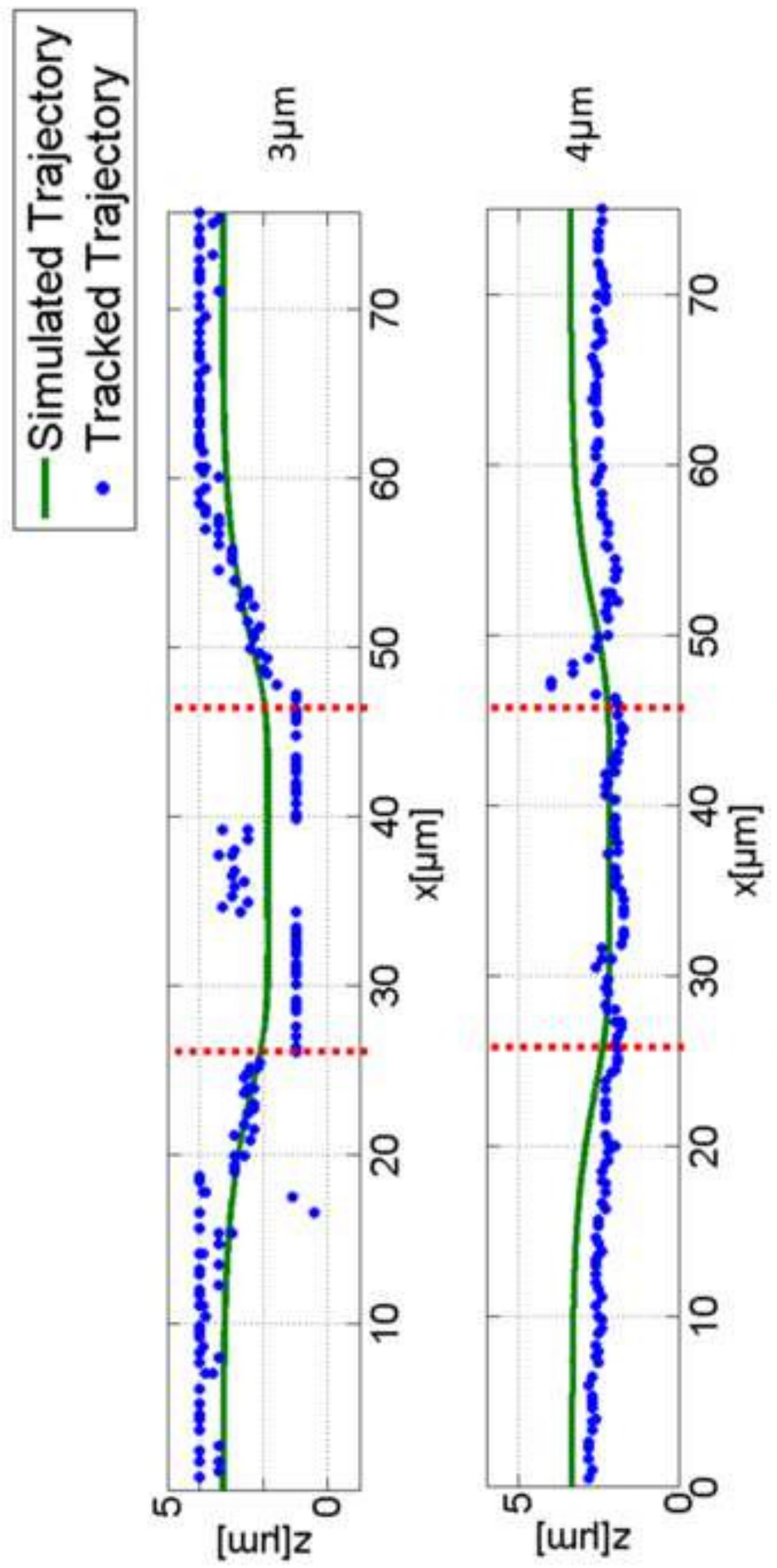

틉

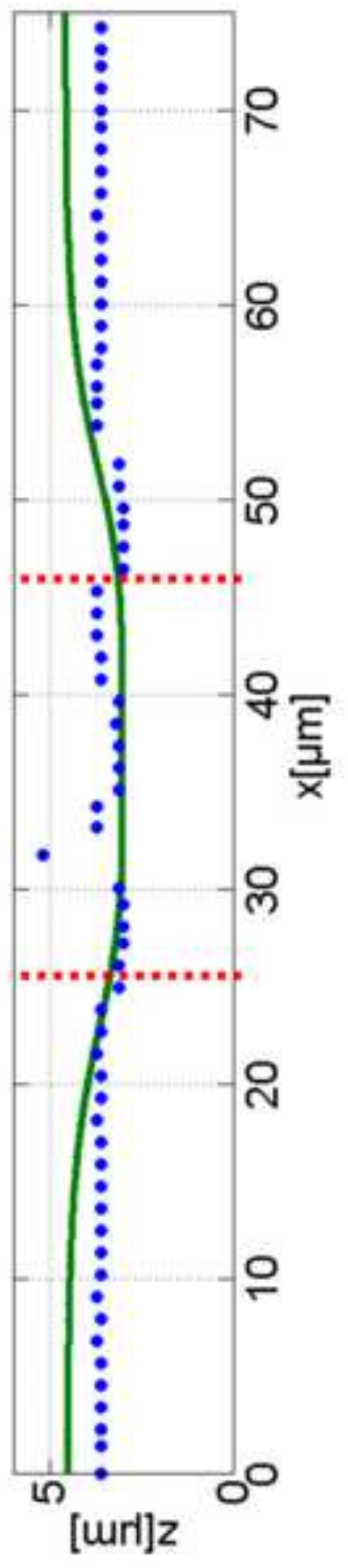

인 


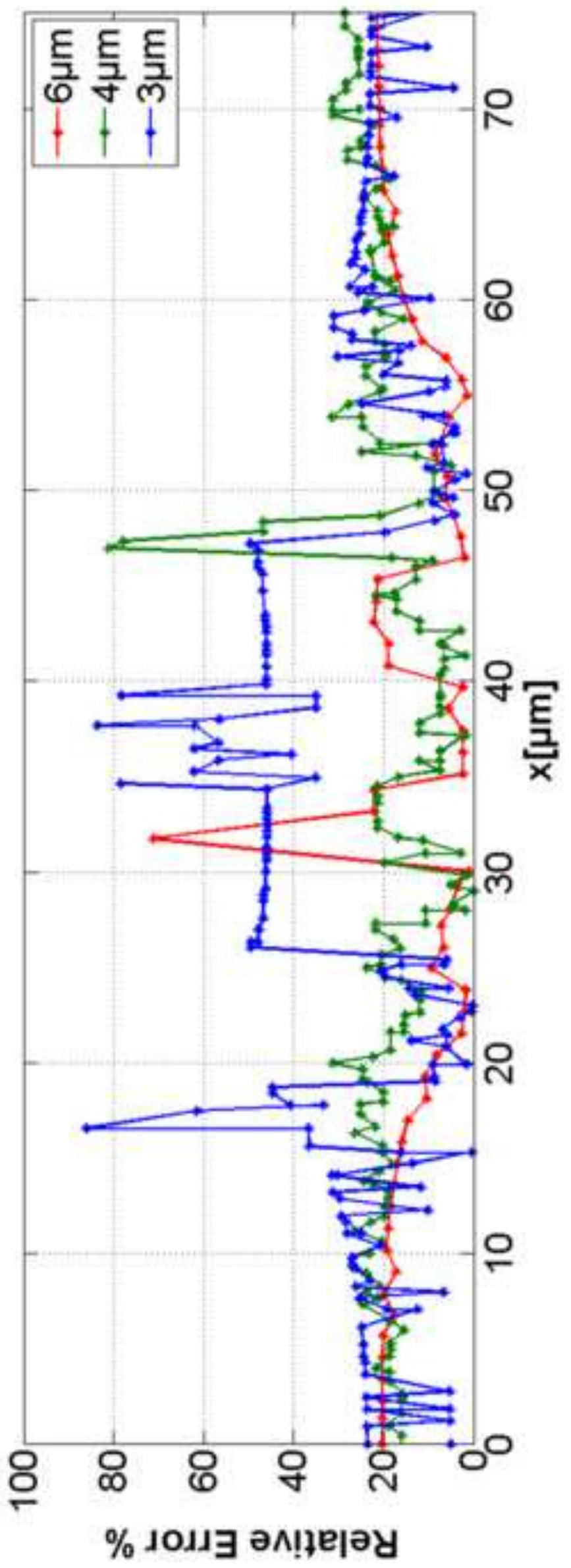

